\title{
108
}

\section{Towards a method for user interface specification}

\author{
Paul Chesson \\ Department of Computer Science \\ The University of Melbourne \\ Parkville, Australia, 3052 \\ email: chesson@cs.mu.OZ.AU
}

\begin{abstract}
A major aspect of user interface specification is concerned with describing the structure of dialogue between an interactive system and its users. The goal of this work is to establish an effective and usable language and process by which the behaviour of a user interface can be completely and accurately defined. This method should enable the production and maintenance of well-structured, understandable, and concise specifications for a wide variety of systems. It should also support specification at multiple levels of abstraction and allow refinement between these levels.
\end{abstract}

KEYWORDS User Interface, Dialogue Specification, Requirements

\section{Introduction}

\subsection{Motivations}

A major aspect of user interface specification is concerned with describing the structure of dialogue between an interactive system and its users. Specifications produced from this process aim to facilitate the communication of requirements and design decisions between the participants in software development. In addition, the user interface specification enables developers to verify that any required constraints are maintained and to analyse various aspects related to the usability of an interface.

There is a need for a universal notation which is adaptable for use for multiple views of the interface behaviour, thereby increasing the scope of its use within software development (Bass, 1994). When applying notations to particular specification problems, rarely is any procedural guidance given. Such guidance would reduce the time needed to learn the language, and avoid the language being used inappropriately (Monk et al., 1993).

\subsection{Proposed objectives}

The goal of this work is to establish an effective and usable language and process by which user interfaces can be completely and accurately defined.

Development of a language suitable for user interface specification should aim to meet the following objectives:

- Communication and Expression

- allow easy understanding of the notation and straightforward translation into an informal description,

- enable the expression of requirements which cover a wide range of levels from the physical actions of a user to the goals which they aim to achieve, and

- provide the ability to express temporal relations, including sequencing, repetition, order independence, interruptibility, mutual interleavibility and concurrency.

- Construction

- facilitate the re-use of parts of a specification in the same system or other systems, where the same or similar functionality is required, and 
- allow parts of the specification to be smoothly coupled with or de-coupled from other parts of the system or other systems.

- Reasoning and Analysis

- enable the resolution of questions related to the user's ability to reach a state or effect from various points within the dialogue space, and

- provide potential to perform various types of usability analysis on a specified interface, including issues such as efficiency, complexity, cognitive consistency, and likelihood and consequences of user errors.

The development of a well-defined method for deriving the specification from a set of informal requirements should address how the following will be achieved:

- production and maintenance of a well-structured, understandable, and concise specification for a wide variety of problems,

- minimisation of the impact of decision-making and the amount of work needed from software developers in the specification process,

- support for task level abstractions,

- the use and mixing of multiple levels of abstraction, whilst maintaining an integrated and coherent specification, and

- refinement between between different levels of abstraction.

\section{Method for language development}

Development of a user interface specification language commenced with the investigation of various existing languages, and their application to a case study of a graphical drawing application. A set of characteristics and capabilities of user interface specification languages was derived for use in assessing and comparing the potential of languages. Various ways of classifying user interface specification languages were also investigated, in order to identify major paradigms for reasoning about and presenting specifications.

The language which was developed aimed to adequately address as many of the characteristics and capabilities as possible, as well as being able to support multiple paradigms to a significant degree. The central objectives were to allow a strong mapping between the components of the language and the natural language requirements with which they corresponded, and a low coupling between components in order to promote ease of modification of an existing specification.

In order to produce formal specifications, the language used to produce a specification must have formally defined semantics. The language under development was given formal semantics by developing a well-defined set of steps which are able to translate any given specification written in the language into a language or notation which has an established set of syntax and semantics.

The initial focus of development was on the lower levels of interaction. The next step was to move towards the specification of user tasks, paying particular attention to the way in which the state of a task is maintained from the history of operations performed. A graphical file manager was used as a test case for this step.

\section{Results}

This section describes the work performed towards the outlined objectives.

\subsection{Case study}

Investigation of various user interface specification languages yielded a list of useful characteristics and capabilities found in such languages (Chesson and Johnston, 1996b), the most important of which became objectives in the development of the new language (see section 1.2).

A case study of how well various languages were able to demonstrate such capabilities proceeded using the requirements for a core piece of functionality from the xfig drawing package. The languages which have been examined are Jacob's language for direct manipulation interfaces (Jacob, 1986), Interactive Cooperative Objects (Palanque et al., 1993), the CNUCE interactor model (Paternó and Faconti, 1992), and Dialogue Specification Notation (Curry and Monk, 1995).

Some of the observations which were particularly relevant to the development of the new user interface specification language include:

- there are dilemmas in choosing between different mechanisms within a language, which can achieve 
the same result,

- there are difficulties in specifying the behaviour of an interface without concern for the details of how this behaviour could be expressed,

- a poor design of framework or model will force developers to have to "work around" the model, rather than with it,

- specifying strong modalities in an interface, whereby the majority of user operations are ignored or have their normal behaviour suspended, can be difficult, and

- handling the creation and deletion of dynamic objects is a significant issue.

\subsection{Classification of languages}

It was found useful to consider languages in terms of how their individual components (states, variables, objects, user events, system actions, etc.) are integrated into a specification. Such paradigms may also closely relate to the way in which the participants in software development read or construct specifications. The following paradigms have been proposed:

State-based paradigm which expresses systems in terms of the status of the interface.

Event-based paradigm which expresses systems in terms of the reactive behaviour of the interface and the changes which may occur.

Grammatical-based paradigm which expresses systems in terms of a top-down hierarchy of operations.

Logic/Constraint-based paradigm which expresses systems in terms of what can be performed and the constraints to be maintained.

Further classification may be achieved by examining the components themselves and the abstractions they represent. For example, objects may represent active agents, visual objects, or conceptual objects in the application domain.

\subsection{Development of FLUID}

A language for specifying user interfaces, FLUID (Formal Language for User Interface Dialogue) (Chesson and Johnston, 1996a), has been developed. The language draws upon logic-based, event-based, and state-based paradigms. The rationale was to provide a wide range of views from which the specification can be read, including those which emphasise logical constraints, hierarchical control and data flow, and the visual status of an interface.

The central unit of abstraction in a FLUID specification is the requirement. Requirements may be elicited from a customer, or included as a result of user interface design decisions. Requirements are expressed declaratively by the use of natural language, allowing the specification to be presented, constructed, and maintained in an intuitive manner.

Individual requirements are related by a hierarchical structure which defines how each requirement constrains and is constrained by others. The dynamic behaviour of the interface is modelled by the propagation of events through this hierarchy.

The behaviour of each requirement is expressed procedurally. This expression is achieved through a combination of mechanisms, namely, the processing of events through the hierarchy, the states of the interface, and the triggering of actions. The mechanisms are provided though a combination of tables and simple coloured Petri nets.

The formal semantics for FLUID have been defined by a process which translates an arbitrary specification written in FLUID into coloured Petri nets. The various graphical components of FLUID are expressed using a set theoretic model whose constraints are also expressed using set theory. This model can then be translated into another set theoretic model which corresponds to a coloured Petri net with inhibitor arcs and priorities. This translation can be performed automatically using a well defined set of steps described in set theory.

The final Petri net generated by the translation process not only enables a precise definition of the behaviour of specifications for interactive systems described in FLUID, but also provides a basis for assisting software developers of such systems. Direct execution of the Petri net will enable validation of the system, and formal techniques may also be applied to verify properties of the interface being developed (Palanque and Bastide, 1995).

\subsection{A framework for requirements}

A three-layer specification model was proposed to provide a framework for the classification and organisation of requirements and their associated FLUID components: 
The symbolic layer deals exclusively with concepts within the user interface environment itself, and excludes any reference to the domain of the application being specified.

The semantic layer deals with simple operations presented by the used, and presented within the context of the application domain.

The pragmatic layer deals with task which are able to be performed by the user, having a practical purpose in performing a piece of work within the context of the application domain.

This model is adopted by FLUID through the use of three constraint hierarchies, connected to each other via the events transferred between them. A set of guidelines describes how events and actions from a specification are used within a particular layer of the model.

High level user tasks and operations can be refined into a "chain" of constraint sub-dialogues which control access to the piece of functionality performed by the task.

\section{Further work}

Development of a method for user interface specification with FLUID will continue by examining natural language requirements from one or more case studies and classifying them according to their abstraction level, purpose, and effects. The purpose of a requirement may be to provide the user with access to the functionality of the application or to constrain such access. The effects of a requirement may relate to its interaction with the environment of the user, or the application.

Each requirement will then be specified through one or more alternative sub-dialogues, each of which satisfies the requirement. Frequently used strategies and steps used to specify particular classes of requirements (as determined in the previous stage), will determine hypotheses for rules which can be applied to other interfaces. Affirmation of this work will be carried out by testing the proposed method on new problems. A set of subdialogues will be established for common forms of interaction in user interfaces.

A similar process will be used to refine and extend requirements down through multiple levels of abstraction, using the three-layer model as a starting point.

\section{References}

Bass, L. (1994). Working group on formal methods in $\mathrm{HCI}$ and software engineering. In Taylor, R. and Coutaz, J., editors, ICSE'94 Workshop on SE-HCI: Joint Research Issues, pages 14-16, Sorrento, Italy.

Chesson, P. and Johnston, L. (1996a). FLUID: Specifying data flow and control for user interfaces. In Yong, L., Herman, L., Leung, Y., and Moyes, J., editors, Proceedings of the First Asia-Pacific Conference on Computer Human Interaction, pages 171-180, Singapore.

Chesson, P. and Johnston, L. (1996b). Objectives for user interface specification languages. In Proceedings of the First Australian Requirements Engineering Workshop, 10 pages, Melbourne. Department of Information Systems, Monash University.

Curry, M. and Monk, A. (1995). Dialogue modelling of graphical user interfaces with a production system. Behaviour and Information Technology, 14(1):41-55.

Jacob, R. (1986). A specification language for directmanipulation user interfaces. ACM Transactions on Graphics, 5(4):283-317.

Monk, A., Curry, M., and Wright, P. (1993). Why industry doesn't use the wonderful notations we researchers have given them to reason about their designs. In Gilmore, D., editor, User-centred requirements for software engineering, pages 253-261, Berlin. Springer-Verlag.

Palanque, P. and Bastide, R. (1995). Verification of an interactive software by analysis of its formal specification. In Proceedings of the IFIP Human-Computer Interaction Conference (Interact'95), pages 191-197, Lillehammer, Norway.

Palanque, P. et al. (1993). Design of user-driven interfaces using petri nets and objects. In Bodard, F., Rolland, C., and Cauvet, C., editors, Conference on Advanced Information System Engineering CAISE'93, pages 569585, Paris, France. Springer Verlag, LNCS n.685.

Paternó, F. and Faconti, G. (1992). On the use of LOTOS to describe graphical interaction. In Monk, A., Diaper, D., and Harrison, M., editors, $\mathrm{HCl}^{\prime} 92$, People and Computers VII, pages 155-173, London. Cambridge University Press. 